\title{
RELATIONSHIP BETWEEN EL NIÑO SOUTHERN OSCILLATION INDEX AND RAINFALL (QUEENSLAND, AUSTRALIA)
}

\author{
G.A. TULARAM \\ Faculty of Science Engineering Environment and Technology (SEET), Griffith University, Australia.
}

\begin{abstract}
This paper explores the relationship between El Niño Southern Oscillation Index (SOI) and rainfall in South East Queensland. Several statistical models of the SOI and rainfall data are analysed including interactions. The results of the analyses show that although the SOI and rainfall can be predicted for short terms using time series analysis methods, mean squared error (MSE) and Theil's U showed that in comparison to persistence model forecasts may not be significantly better. Although the short term predictions may be worse, this does not mean that these cannot be used; rather, it shows that for different fitting and forecast periods, models can fail in predicting ahead. In fact weather forecasts can predict the short term rainfall well - but cannot predict monthly precipitation for two or three or more months ahead. In climate predictions the limit of predictability is reached when the standardised MSE reaches the climate variance $(=1)$. All the rainfall models used in this study show an MSE well below 1, i.e. the models did not reach the limit of predictability.
\end{abstract}

Keywords: El Niño, predicting rainfall, rainfall modelling, SOI, time series.

\section{INTRODUCTION}

The El Niño Southern Oscillation (ENSO) is an important climate phenomenon that affects primarily the atmospheric conditions of the tropical Pacific region including the local climate and weather in Australia. The ENSO is measured by the SOI, the Southern Oscillation Index. The SOI is basically the monthly averaged pressure difference between Darwin and Tahiti. A number of authors have researched the relationship in the past using different approaches to model the ENSO. An attempt is made here to apply time series analysis and forecasting methods to predict rainfall using SOI.

Katz [1] reviewed the history of statistical modelling of the SOI, while Fedorov et al. [2] reviewed the physical predictability of the ENSO. Both concluded that the ENSO may not be predictable so far by deterministic physical models, and they rather prefer probabilistic forecasts based on ensemble forecasts. In 2005, Ahn and Kim [3] modelled the SOI by using nonlinear univariate time series models. They found that the SOI time series model can be simulated by a combination of autoregressive and Garch models. Whereas the ENSO is a physical process and therefore complicated, Latif et al. [4] and Neelin et al. [5] argued that prediction of the effects on rainfall may be described statistically.

How ENSO affects tropical areas and especially Australia has been examined as well. In 1998, Chiew et al. [6] showed that dry conditions in Australia might be associated with El Niño. They concluded that although ENSO and rainfall in Australia are connected to each other, this link is 'not sufficiently strong to consistently predict the hydro-climate variables accurately'. Murphy and Ribbe [7] examined South East Queensland rainfall and its connection to ENSO and stated that the South East Queensland rainfall is highly inter-decadal variable with longer dry periods and extreme wet periods. It seems that the variability of rainfall in northern areas of Australia might be linked to the ENSO. Furthermore ENSO appears to affect areas that depend on the rainfall, e.g. fruit production which has been shown by Wright et al. [8] and wheat yield forecasts examined by Podbury et al. [9]. Therefore, it would be useful to be able to predict the ENSO and thus forecast its affects. 
Climate change is an important current issue and it may have an impact on the ENSO. Timmermann [10] examined how the global greenhouse warming affects the stability of ENSO. Although scientists have made efforts on climate prediction, the results cannot be used to predict the SOI and its effects directly. The recent drought conditions in Queensland and Australia have led to government restrictions on water usage. Hence, it would be important to examine SOI, rainfall and their interrelationship. This paper attempts to provide a better understanding of the modelling of SOI and monthly precipitation in South East Queensland using time series methods. The study also analyses predictive capacities of various time series models. The insights gained regarding the interaction between the SOI and rainfall will help improve models generally.

\section{METHOD}

In the main, the time series (linear) regression and modelling procedures are used to study and analyse the large datasets. However, nonlinear exponential regression is also utilised. The SOI dataset was provided by the Climate Prediction Centre of the (American) National Weather Service. The Bureau of Meteorology (Australia) also holds rather large datasets of high quality climate and weather data. The rainfall data were acquired from the bureau. The SOI included monthly data from 1951 to 2007. The rainfall data were based on monthly rainfall figures gathered in a station in Beaudesert (QLD) which is $40 \mathrm{~km}$ south of Brisbane approximately the same distance inland from the Gold Coast.

\subsection{SOI}

SOI is a measurement for the ENSO and calculated by subtracting the long term average air pressure difference between Darwin and Tahiti from the monthly average air pressure difference and then dividing it by the standard deviation of the long term air pressure difference:

$$
\mathrm{SOI}=\frac{\Delta P-\Delta P \text { avg }}{{ }^{s} \Delta P}
$$

The measure may be a positive or negative. The positive SOI indicates an El Niño event, which is of interest in this paper. Figure 1 shows the time series of the SOI for the period. The minimum SOI value noted was -4.6 while the maximum value was 2.9 . The data also provided useful information about the range. The overall mean value of SOI was calculated to be -0.1722 , the median -0.2 and the standard deviation 1.084 .

\subsection{Rainfall data for Beaudesert (QLD)}

The dataset for rainfall was rather large and dated from May 1892 to May 2007. However, it had four missing values and it was decided to fill them with an average value (average of the rainfall 1 month before and after). The mean rainfall was noted to be $52.60 \mathrm{~mm}$ per month with a minimum of 0 and a maximum of $600.40 \mathrm{~mm}$ per month. The standard deviation was $72.65 \mathrm{~mm}$ per month that indicates a highly variable rainfall. Figure 2 shows the rainfall time series for the period indicated above.

There are several statistical models used in this study. These are explained in the following paragraphs.

\subsection{Linear regression}

Linear regression models the relationship between two variables. The model equation can be written as

$$
Y=c+\alpha X+\epsilon
$$




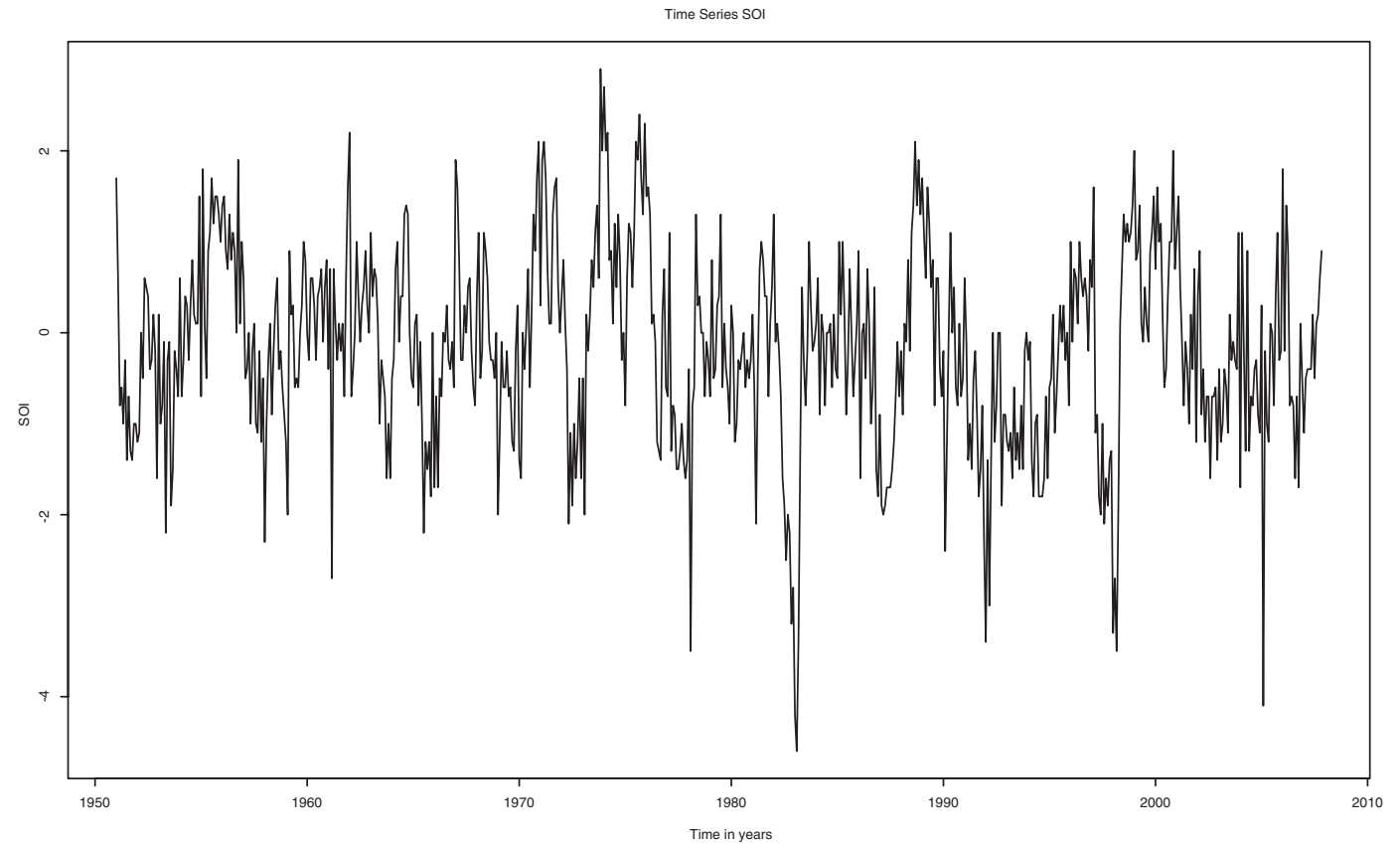

Figure 1: Monthly SOI data (1951-2007).

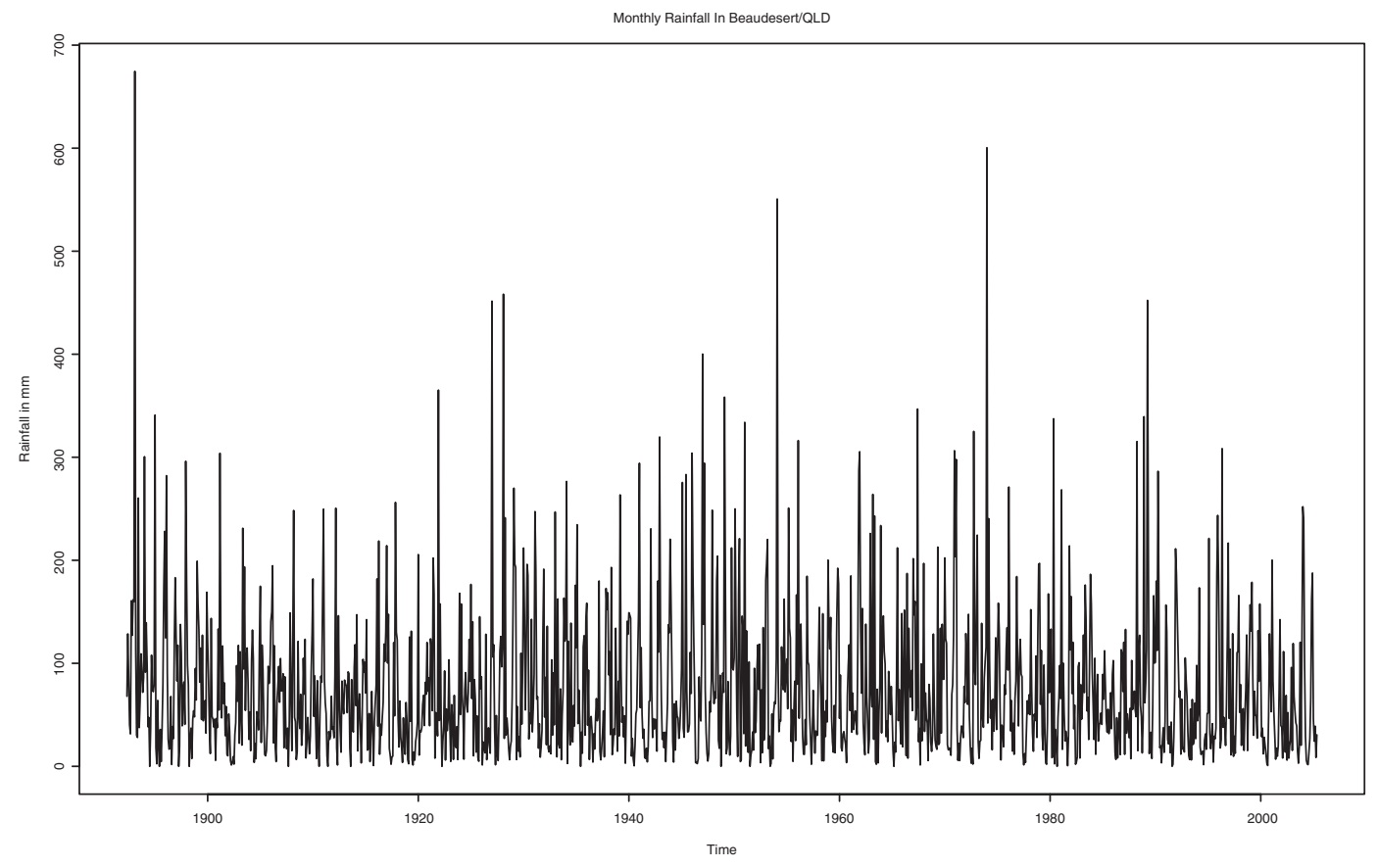

Figure 2: Monthly rainfall (mm) for Beaudesert (QLD) (1852-2007). 
where $a$ is the slope, $c$ is the intercept, and $\epsilon$ denotes the residual. The residual or error term is identically and normally independently distributed. By transforming the variables, the linear regression method can also be used to fit nonlinear models and statistical codes can be written for packages that can perform the fitting for such models. To choose the best regression model it is useful to have a look at the correlation coefficient and tests for statistical significance. It is important to study the residuals to determine whether it is identically and independently distributed.

\subsection{SARIMA}

ARIMA stands for autoregressive integrated moving average and it is a combination of autoregressive (AR) and moving average (MA) models. It considers non-stationary data with the term 'integrated' standing for differencing of the data to achieve stationarity.

Generally ARIMA is given in the notation

$$
\operatorname{ARIMA}(p, d, q)(P, D, Q)_{s}
$$

where $p$ and $q$ are the order of the $\operatorname{AR}(p)$ and $\operatorname{MA}(q)$ model, $d$ is the order of differencing at lag $1, s$ is the length of one season (period), $P$ and $Q$ are the order of the seasonal $\operatorname{AR}(P)$ and seasonal $\mathrm{MA}(Q)$ model and $\kappa D$ is the order of seasonal differencing. In the backwards notation, the model can be written like this:

$$
\begin{aligned}
& \left(1-B^{s}\right)^{D}(1-B)^{d}\left(1-B_{\varphi 1}-\cdots-B^{p} \varphi_{p}\right)\left(1-B^{s} \Phi_{1}-\cdots-B^{s P} \Phi_{p}\right) Y_{t} \\
& \quad=\left(1-B \theta_{1}-\cdots-B^{q} \theta_{q}\right)\left(1-B^{s} \Theta_{1}-\cdots-B^{s Q} \Theta_{Q}\right) e_{t}
\end{aligned}
$$

with $\varphi_{j}$ as the $j^{\text {th }}$ autoregressive parameter, $\Phi_{j}$ as the $j^{\text {th }}$ seasonal autoregressive parameter, $\theta_{j}$ as the $j^{\text {th }}$ moving average parameter and $\Theta_{j}$ as the $j^{\text {th }}$ seasonal moving average parameter.

Autoregressive processes are a linear regression on the past $(p)$ values of the time series itself. Moving average models rely on the past residuals or past $(q)$ error terms. In general, a first guess of the model order is done by evaluating the autocorrelation (ACF) and partial autocorrelation (PACF) functions of the time series. The ACF and PACF can also be used to check for stationarity although it is better to apply a unit root test. Automatic routines to find the model order often try to minimise the squared error or the AIC, respectively, to maximise the log-likelihood value (Makridakis et al. [11]).

A basic assumption for sARIMA models is that the data are covariance-stationary.

\section{$2.5 \mathrm{GARCH}$}

Although seasonal ARIMA models are covariance-stationary, GARCH (generalised autoregressive conditional heteroskedasticity) models are used to analyse volatility. A GARCH model can be written as

$$
\begin{gathered}
y_{t}=C+\epsilon_{t} \\
\sigma_{t}^{2}=\sum_{j=1}^{p} \gamma_{i} \sigma_{t-i}^{2}+\sum_{j=1}^{q} \alpha_{j} \epsilon_{t-j}^{2}
\end{gathered}
$$

Equation (5) is known as the mean equation and eqn (6) the conditional variance equation. It is a function of ARCH $\left(\epsilon_{t-j}^{2}\right)$ and GARCH $\left(\sigma_{t-i}^{2}\right)$ terms. $p$ and $q$ define the order of the model. Usually $\rho$ and $q$ are chosen to be 1 (Password [12] and Zhou et al. [13]). 


\subsection{GARCH in mean (GARCH-M)}

When the volatility influences the mean equation, an extra term is added:

$$
\begin{gathered}
y_{t}=C+k \sigma_{t}+\epsilon_{t} \\
\sigma_{t}^{2}=\sum_{j=1}^{p} \gamma_{i} \sigma_{t-i}^{2}+\sum_{j=1}^{q} \alpha_{j} \epsilon_{t-j}^{2}
\end{gathered}
$$

where $k$ denotes the influence of the volatility in the mean equation.

\subsection{ARMA/GARCH}

The mean equation can be replaced by an ARMA model to get a combination of ARMA and GARCH models. The mean is modelled by an autoregressive moving average model. The equations become:

$$
\begin{gathered}
\left(1-B \varphi_{1}-\cdots-B^{p} \varphi_{p}\right) Y t=\left(1-B \theta_{1}-\cdots-B^{q} \theta_{q}\right) e_{t} \\
\sigma_{t}^{2}=\sum_{j=1}^{p} \gamma_{i} \sigma_{t-i}^{2}+\sum_{j=1}^{q} \alpha_{j} \epsilon_{t-j}^{2}
\end{gathered}
$$

In fact, if one decides to use differenced data, even ARIMA models are possible. Clearly many combinations of models are possible.

\section{RESULTS}

The analysis involved splitting of the datasets; a testing period and a fitting period. The fitting period covered the last 24 months of the datasets. At first, the fitting period is used to search for the best models; and secondly, those models are compared by forecasting the test period. In the end, all the models are tested by examining forecasts for longer periods. The fitting and testing periods are then changed and the models were refitted. From those results, mean forecasting measurements are obtained, which provide a general idea about the forecasting quality of the models found. The models that were found by automatic procedures in the R statistical package were also included in the analysis. The Theil's U statistic and the mean squared error (MSE) were used as quality indicators.

\subsection{SOI}

At first the SOI is modelled directly with the time series methods defined above. The DickeyFuller test applied to the SOI showed that the SOI is a stationary time series. The PACF and ACF of the time series are used to estimate the order of ARIMA. The PACF and ACF can be seen in Fig. 3. The PACF suggests an AR(3) model, the ACF here is typical for an $\operatorname{AR}(p)$ model.

The few spikes at larger lags may show some seasonality. The described spikes at 2 years in the PACF may lead to a seasonal AR(2) process, which is added to the AR(3) process. Interestingly, an automatic fit (by searching the lowest AIC) suggests an $\operatorname{ARIMA}(2,0,2)$ model. 


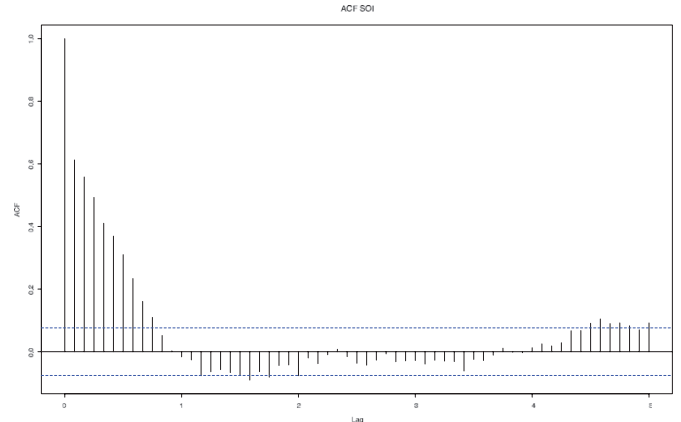

(a)

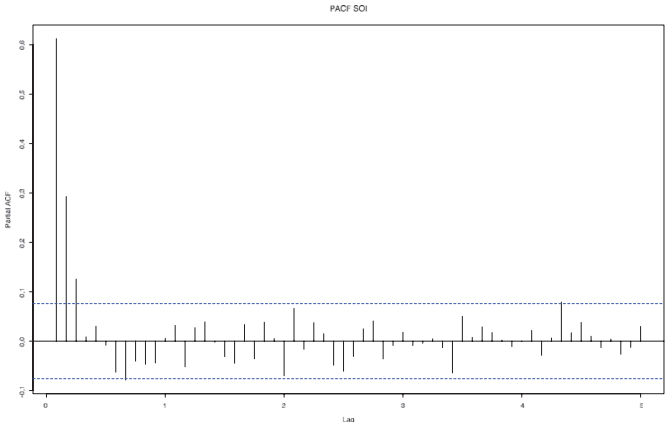

(b)

Figure 3: The (a) ACF and (b) PACF of the SOI.

Table 1: MSE, AIC and the log-likelihood values for those three models.

\begin{tabular}{lccc}
\hline & ARIMA(3,0,0) & sARIMA $(3,0,0)(2,0,0)_{12}$ & ARIMA $(2,0,2)$ \\
\hline MSE & 0.6540 & 0.6485 & 0.6471 \\
AIC & $1,601.06$ & $1,599.6$ & $1,596.12$ \\
Log-likelihood & -795.53 & -792.8 & -792.06 \\
\hline
\end{tabular}

Table 2: MSE and U values of forecasts 6 month ahead.

\begin{tabular}{lccc}
\hline & ARIMA $(3,0,0)$ & SARIMA $(3,0,0)(2,0,0)_{12}$ & $\operatorname{ARIMA}(2,0,2)$ \\
\hline MSE & 1.087339 & 0.995922 & 0.9491391 \\
$\mathrm{U}$ & 0.8584589 & 0.8372988 & 0.7689262 \\
\hline
\end{tabular}

The models are: $\operatorname{ARIMA}(3,0,0)$, then $\operatorname{sARIMA}(3,0,0)(2,0,0)_{12}$, and $\operatorname{ARIMA}(2,0,2)$. Table 1 shows the MSE, AIC and the log-likelihood values. Although all the models perform similar during the training period, they show differences in the forecast 6 months ahead (Table 2).

The model with the lowest AIC performs best, namely the ARIMA(2,0,2)-model. It has the lowest MSE and U values. The residual time series resembles white noise. The ACF and PACF of the residuals do not show any significance. The Box-Pierce test shows that the residuals are not correlated. Therefore it is concluded that the $\operatorname{ARIMA}(2,0,2)$ model may simulate the SOI satisfactorily.

Another approach was to investigate various GARCH models. Briefly, the results do not differentiate much from the ARIMA models. The order $(1,1)$ for GARCH-models has been chosen as it is appropriate to model time series data in this case. The models are compared in this analysis: a pure GARCH(1,1)-M, an AR(8)-GARCH(1,1)-M model and a GARCH(1,1)-M model with automatic ARIMA fit (in this case, it is the previous $\operatorname{ARMA}(2,2)$ model). An AR(8)/ GARCH(1,1)-M model has been chosen because Ahn and Kim [3] found that an AR(8)/ $\operatorname{GARCH}(1,1)$ might be able to predict the SOI well. The fitting period leads to the following results (Table 3 ). 
Table 3: MSE and U values of GARCH(1,1)-M, an AR(8)-GARCH(1,1)-M model and a ARMA(2,2)/GARCH(1,1)-M model.

\begin{tabular}{lccc}
\hline & GARCH(1,1)-M & AR(8)/GARCH(1,1)-M & ARMA(2,2)/GARCH(1,1)-M \\
\hline MSE & 1.174351 & 0.6470886 & 0.6470798 \\
$\mathrm{U}$ & 0.955743 & 0.9386803 & 0.7739873 \\
\hline
\end{tabular}

Table 4: MSE and U for forecasts 6 months ahead.

\begin{tabular}{lccc}
\hline & GARCH(1,1)-M & AR(8)/GARCH(1,1)-M & ARMA(2,2)/GARCH(1,1)-M \\
\hline MSE & 1.335846 & 0.966652 & 0.9467035 \\
$\mathrm{U}$ & 0.9808247 & 0.8005486 & 0.7666965 \\
\hline
\end{tabular}

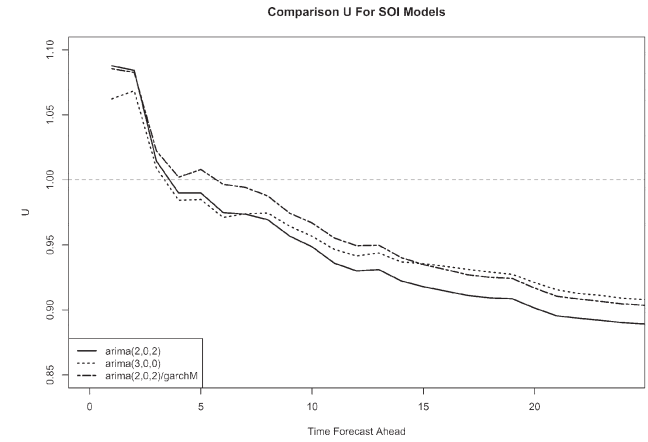

(a)

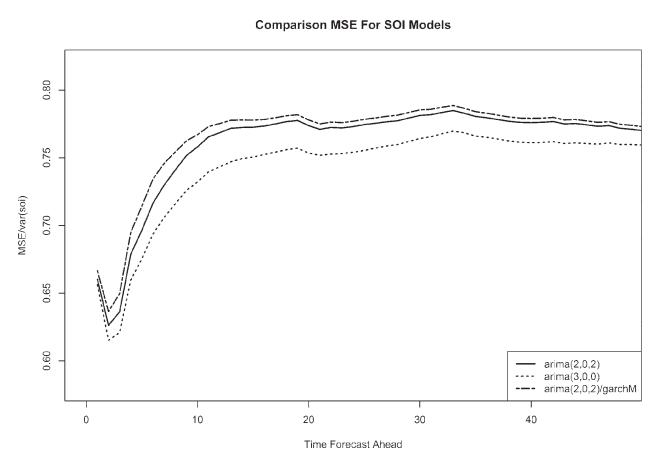

(b)

Figure 4: The (a) mean U and (b) mean MSE of forecasts of the SOI.

A pure GARCH in mean model performs worse than those models that include ARIMA in their equations. The MSE and U do not show a great difference to the ARIMA models used previously even when the MSE and U values are slightly better. The reason is that combined ARIMA/GARCH models model the residuals from the ARIMA fit. If the residuals are already white noise and do not show heteroskedasticity, then it is not surprising that the results did not improve much. The forecast 6 months ahead does not show a great improvement when compared with the ARIMA models. The results (Table 4) show that a pure GARCH model clearly cannot predict the SOI. Here, the forecast 6 months ahead has been done best by the ARMA(2,2)/GARCH(1,1)-M model.

It is known that forecasting results differ with the chosen periods for fitting and testing. Therefore to be able to better judge the quality of the forecasts, the fitting and testing periods have been changed and the model has been fitted repeatedly. Several predictions have been obtained which is used to calculate mean statistics. For example, the forecast one period ahead has been done 80 times, two periods ahead 79 times in order to calculate a mean MSE and a mean U value. Figure 4 shows the mean MSE (standardised by the variance of the SOI) and $\mathrm{U}$ of $\operatorname{ARIMA}(2,0,2), \operatorname{ARIMA}(3,0,0)$, and $\operatorname{ARMA}(2,2) / \mathrm{GARCH}(1,1)-\mathrm{M}$ models for different forecasting periods ahead.

Figure 4a shows that the mean $U$ values depend on the forecast period. In comparison to a naïve forecast, all the models perform worse for short term predictions. Longer term forecasts are better 
than persistence predictions. Compared to persistence, the $\operatorname{ARIMA}(2,0,2)$ model performs best. Nevertheless, the mean MSE values give a better measurement to compare the quality of the models. Figure $4 \mathrm{~b}$ shows that the ARIMA(3,0,0) model has the lowest MSE for different forecasting and fitting periods. These figures show that the used statistical models cannot be used for a short term prediction of the SOI generally - the naïve forecast performs better for different testing periods. For long term predictions the MSE of those models reaches an equilibrium level. The reason is the nature of the used statistical forecasting methods: undifferentiated ARIMA models converge to a constant. Even if a volatility term is added to the mean equation it does not change this behaviour significantly. That is to say it may be problematic to use statistical forecasting methods for the SOI.

Figure 5 shows a 24 months point forecast, its $95 \%$ confidence interval and the observed SOI. It can be seen that for this forecasting period the observed data lie well within the $95 \%$ confidence bounds. For the SOI such a confidence interval is rather large. Clearly, a positive and negative SOI represent different situations (positive SOI is El Niño). The SOI might be simulated by an ARIMA model and the statistical forecast may lead to reasonable error and quality measurements, but the predictions are not physically correct.

\subsection{Rainfall}

The rainfall data were analysed in a similar manner. The rainfall time series was not stationary, therefore needed differencing. After seasonal differencing the dataset was found to be stationary. This was verified by a Dickey-Fuller test, which results in a $p$-value of less than 0.01 . The ACF and PACF of the seasonal differenced data confirm stationarity (Fig. 6). The ACF and PACF of the seasonal differenced rainfall first suggest a seasonal MA(1) model. Nevertheless by minimising the

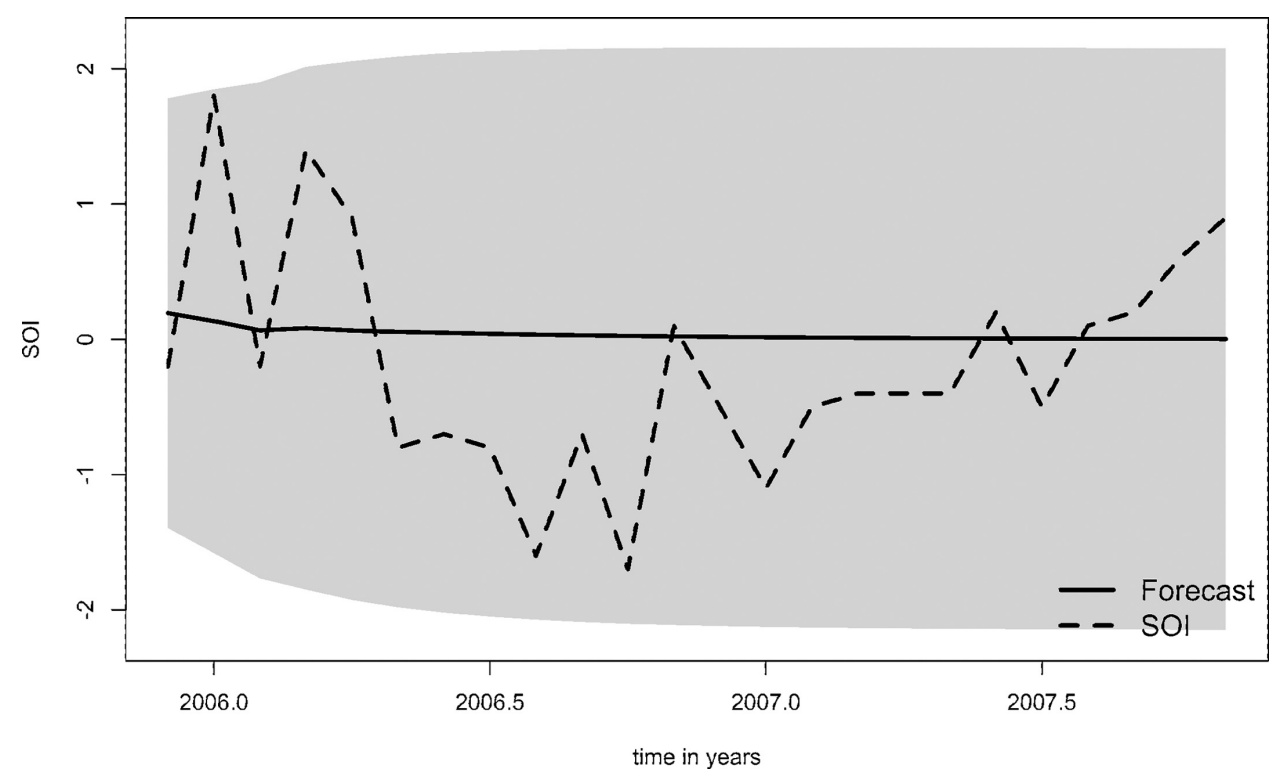

Figure 5: The 24 months forecast of the SOI including the original time series. The shaded area indicates the $95 \%$ confidence interval. 


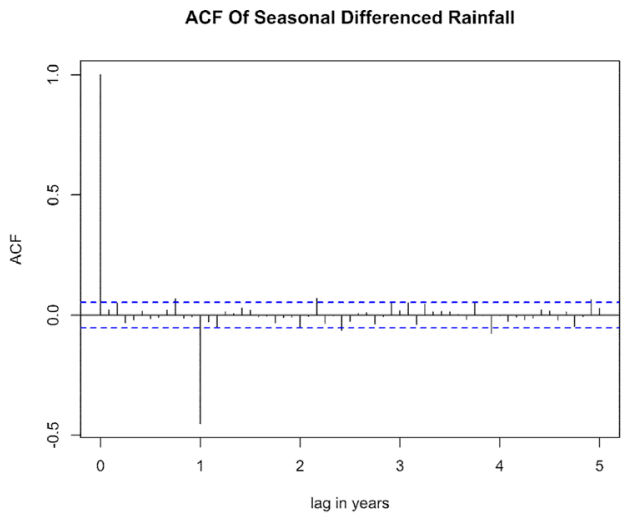

(a)

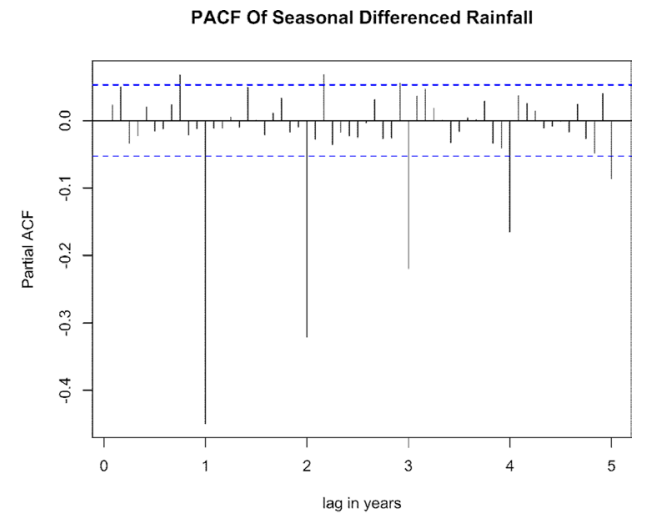

(b)

Figure 6: The (a) ACF and (b) PACF of rainfall.

Table 5: MSE, AIC and log-likelihood for sARIMA models.

\begin{tabular}{lcc}
\hline & SARIMA $(2,0,0)(1,1,1)_{12}$ & SARIMA $(4,0,2)(1,1,2)_{12}$ \\
\hline MSE & 4,400 & 4,385 \\
AIC & $15,166.25$ & $15,170.68$ \\
Log-likelihood & $-7,578.12$ & $-7,575.34$ \\
\hline
\end{tabular}

Table 6: MSE and U values of various GARCH type models.

\begin{tabular}{lcc}
\hline & $\begin{array}{c}\text { sARIMA }(2,0,0)(1,1,1)_{12} / \\
\text { GARCH( }(1,1)-M\end{array}$ & $\begin{array}{c}\text { sARIMA(4,0,2)(1,1,2) }{ }_{12} / \\
\text { GARCH(1,1)-M }\end{array}$ \\
\hline MSE & $4,349.564$ & $4,333.937$ \\
$\mathrm{U}$ & 0.5229821 & 0.6038211 \\
\hline
\end{tabular}

AIC a sARIMA $(2,0,0)(1,1,1)_{12}$ model; and by minimising the MSE a $\operatorname{sARIMA}(4,0,2)(1,1,2)_{12}$ model can be found. Table 5 compares the MSE, AIC and log-likelihood of mentioned models. The residuals of both models are white noise (not shown). Nonetheless, the models fail in describing the variability of the original time series. Therefore GARCH models are applied to the rain time series directly. The previously considered ARIMA models are now extended by GARCH $(1,1)-\mathrm{M}$ models that improve the MSE (Table 6).

In a similar manner to the SOI, mean MSE (standardised by the variance of the rain) and U values were calculated for different prediction periods (see Fig. 7). The tested models are not significantly different in both the MSE and U value. In general such models perform worse than a naïve forecast for short term predictions. This however does not imply that they should not be used for short term predictions. For longer term predictions (greater than 10 months ahead) all models (except $\left.\operatorname{sARIMA}(2,0,0)(1,1,1)_{12}\right)$ are better than persistence. 


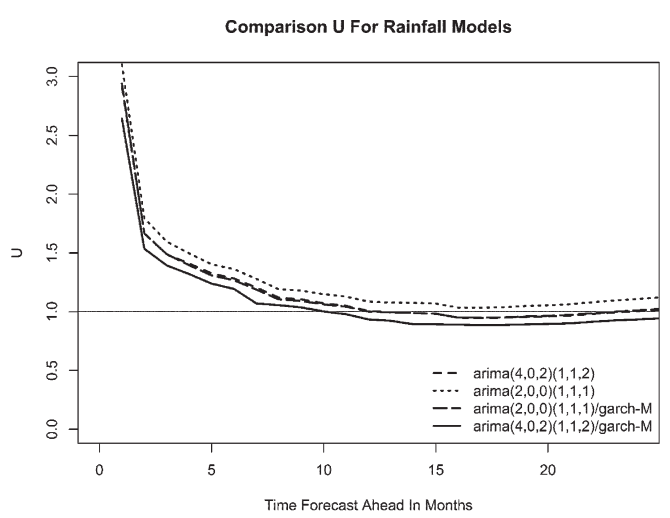

(a)

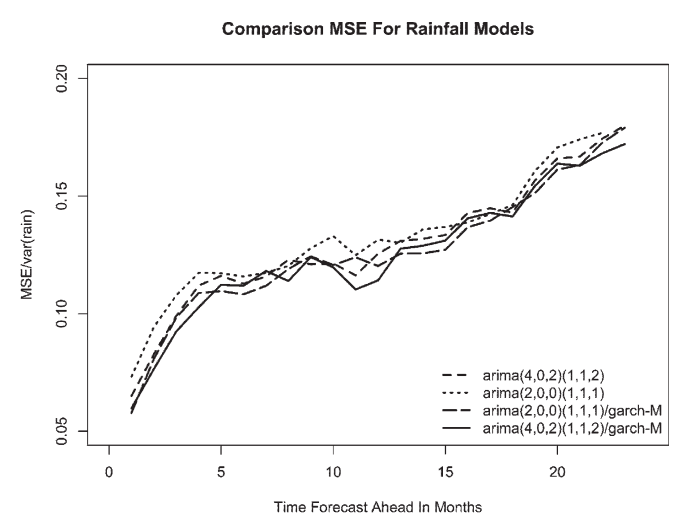

(b)

Figure 7: The (a) mean U and (b) mean MSE of forecasts of rainfall.

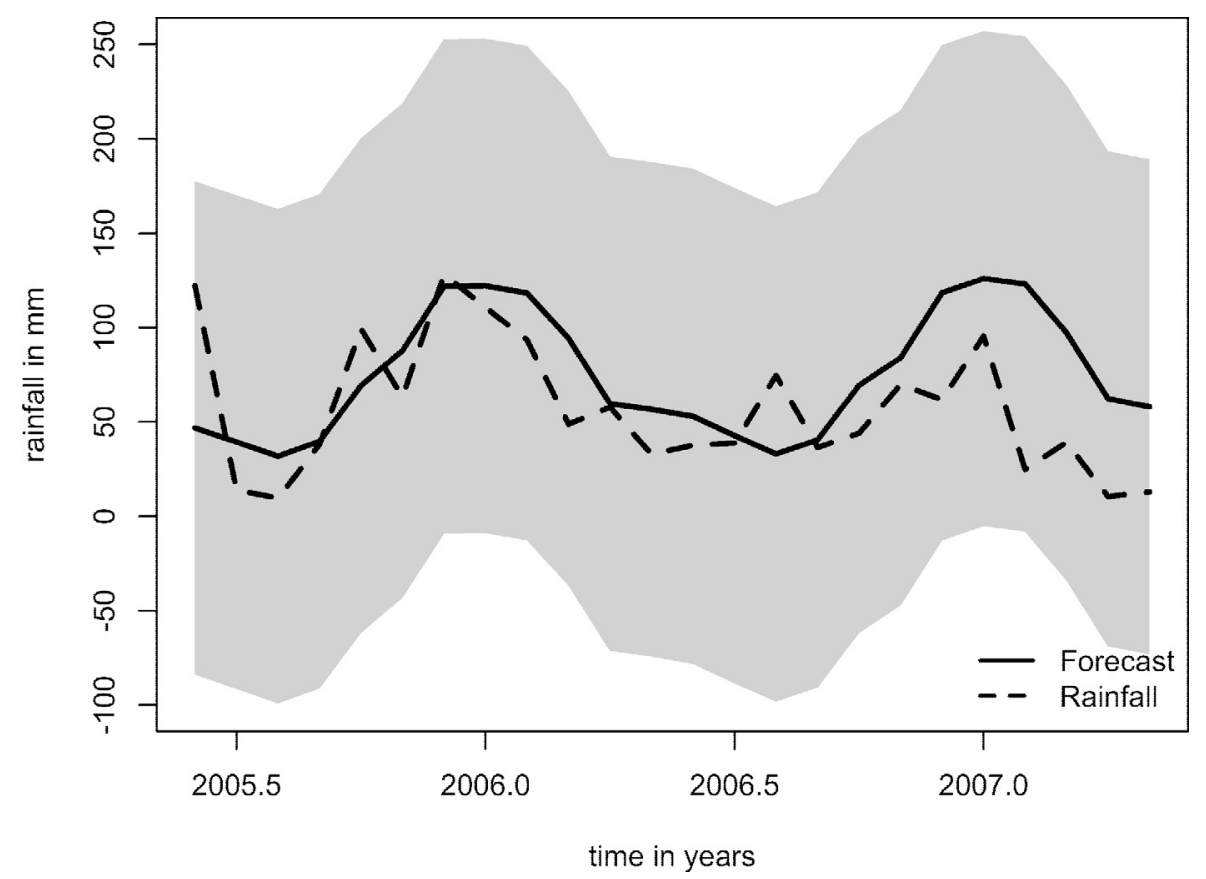

Figure 8: The 24 months forecast of the rainfall at Beaudesert including the original time series. The shaded area indicates the $95 \%$ confidence interval.

Figure 8 shows the 24 months forecast using $\operatorname{sARIMA}(2,0,0)(1,1,1)_{12}$ - the one with the lowest AIC in the actual testing period. The forecast catches the rainfall quite well. It seems that after 16 months the model predicts an amount of rainfall which is too high. This is the period in which South East Queensland started to suffer from the most recent drought. It could be that in these months a new regime for the rainfall took over which had been independent from the previous time 
steps. That might be beyond statistical time series analysis and perhaps (physical) climate modelling and further research is needed.

\subsection{Linear exponential regression}

Rainfall and the SOI are related to each other. The ENSO affects the climate in South East Queensland. Figure 9a shows the cross correlation function of the smoothed SOI and smoothed rainfall (i.e. filtering out high frequencies). It can be seen that the SOI has indeed effects on the rainfall. Since the time windows of the SOI and rainfall are not equal, the datasets have been trimmed so that both cover the period from January 1951 to May 2007. Afterwards all the data from November 2006 are omitted. In this case, 6 months data were held back to evaluate the results.

In such a relationship, a linear regression between the SOI and the rainfall needs to be found. The datasets have not been filtered or smoothed. The scatter plot in Fig. $9 \mathrm{~b}$ does not suggest a strong correlation. Nevertheless exponential linear regression yields the best results with a correlation coefficient of 0.2535 that is statistically significant at 0.01 level. From this result the values from the regression rain $=c+a e^{s o i}+\epsilon$ are fitted and residuals are obtained and shown in Fig. 10. The nonlinear regression model does not reflect the rainfall well. Therefore, the residuals are modelled by a seasonal ARIMA and by an ARIMA/GARCH-M model. For the ARIMA model one needs to assume that these residuals have a stationary variance. After seasonal differencing, the Dickey-Fuller test shows that the residuals are stationary. The ACF and PACF of the residuals shown in Fig. 11 look similar to those from the seasonal differenced rainfall. That is why a seasonal ARIMA model of order $(2,0,0)(1,1,1)$ and an $\operatorname{ARIMA}(2,0,0)(1,1,1) /$ GARCH(1,1)-M were chosen. Obtained residuals appear stationary similar to white noise series for either residual model (not shown). Table 7 compares the types of models and in addition it also provides the results of the exponential regression. The prediction ahead has been done by forecasting the SOI first and the rainfall afterwards. The models that have performed best in the training period appear worse in predicting ahead. Furthermore, it seems that linear (exponential) regression without any residual modelling is better for short term forecasts. Figure 12 shows the mean MSE and U values for different forecasting periods. It is noticeable that neither method

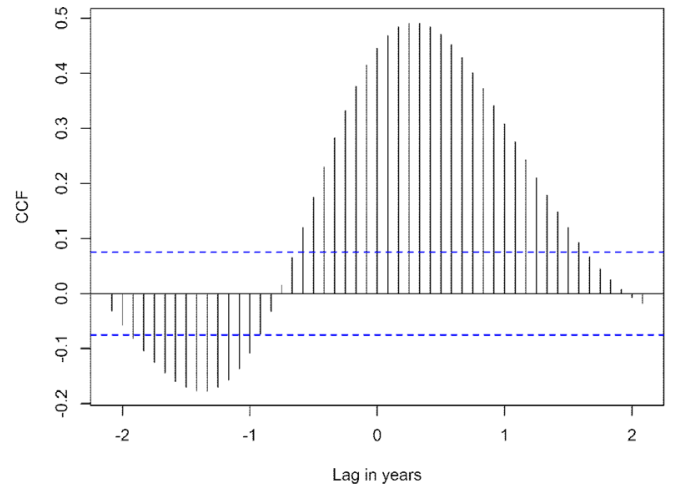

(a)

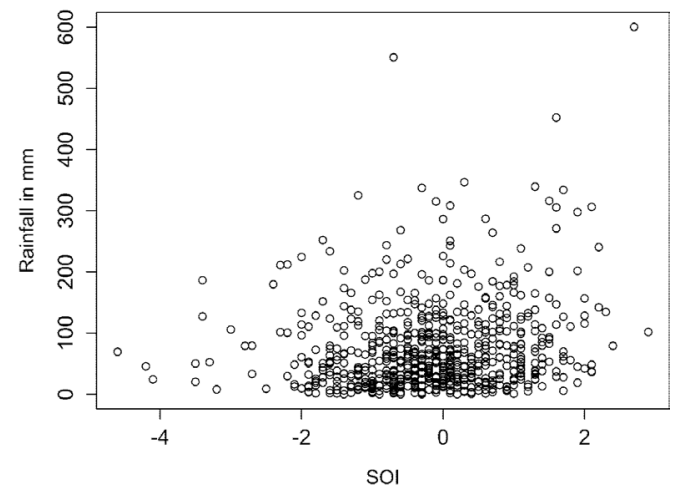

(b)

Figure 9: The (a) cross correlation function of smoothed SOI and rainfall, and (b) scatter plot of the SOI and Beaudesert rainfall. 


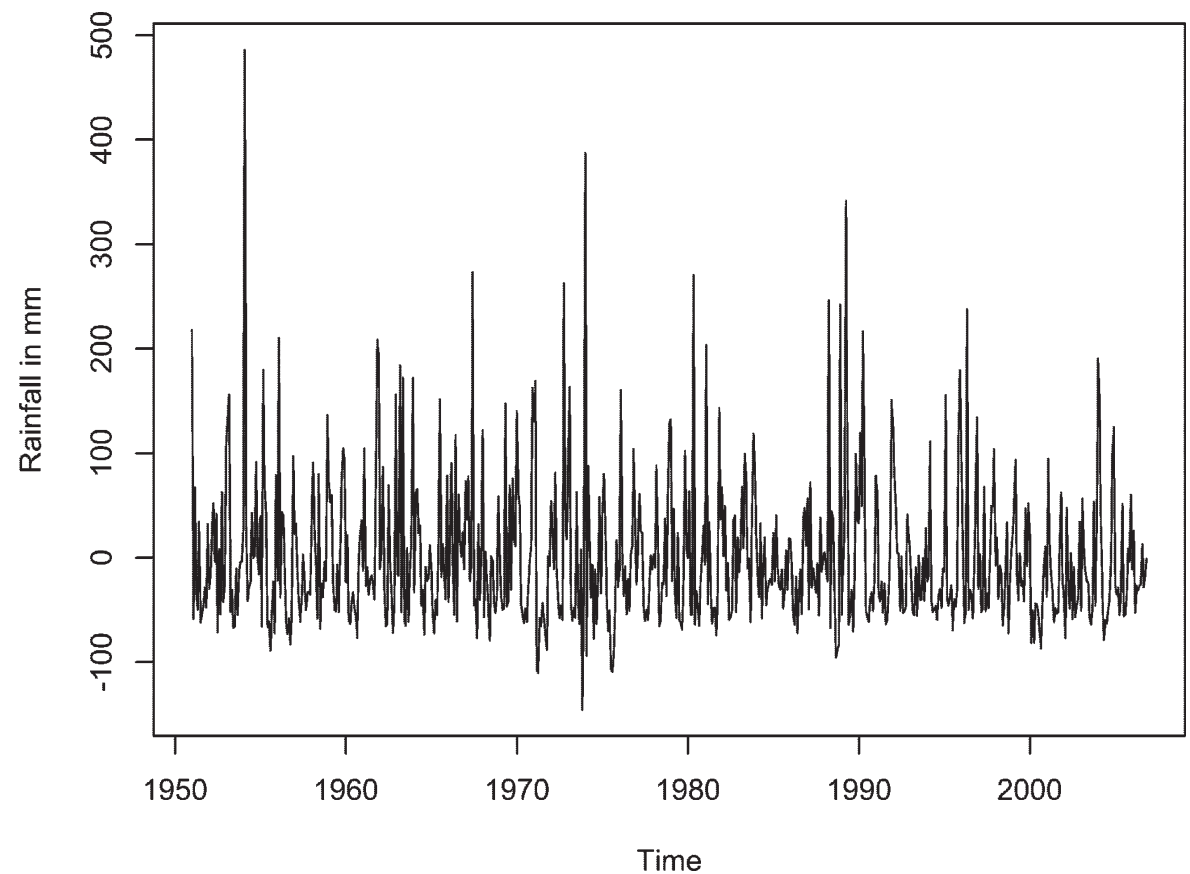

Figure 10: Residuals from exponential regression.

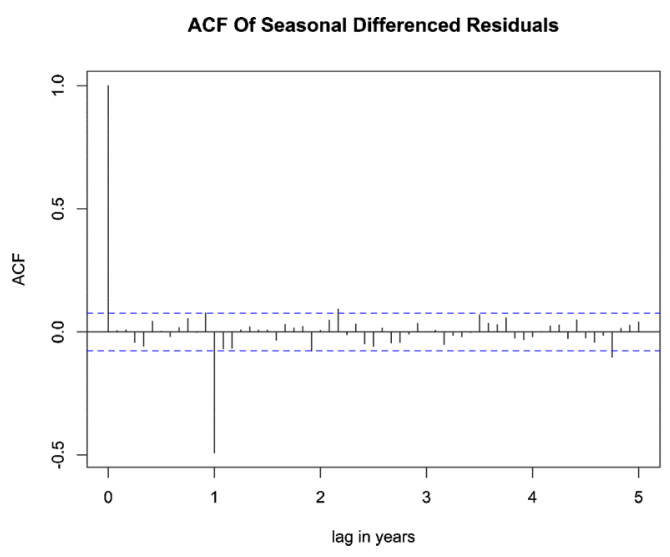

(a)

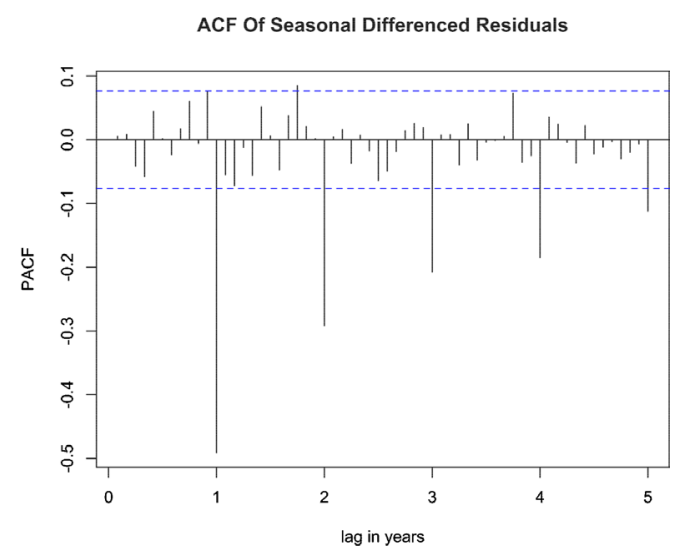

(b)

Figure 11: The (a) ACF and (b) PACF of seasonally differenced residuals from exponential linear regression describing rainfall.

leads to good results compared to persistence. The MSE is lowest for short term predictions for the linear regression combined with ARIMA $(2,0,0)(1,1,1)$. The mean MSE in Fig. 12 is standardised and therefore is comparable to Fig. 7 that showed the mean MSE for time series modelling of the rainfall. The linear regression or ARIMA and GARCH models tend to have approximately the same prediction errors. 
Table 7: MSE and U values for fitted and 3 and 6 month forecasts for the models: (1) linear exponential regression combined with residual modelling via (2) $\operatorname{sARIMA}(2,0,0)$ $(1,1,1)_{12}$ and (3) GARCH-M(1,1) additionally.

\begin{tabular}{lcccccc}
\hline $\begin{array}{c}\text { MSE for fitted } \\
\text { values }\end{array}$ & $\begin{array}{c}\text { U for fitted } \\
\text { values }\end{array}$ & $\begin{array}{c}\text { MSE for } \\
\text { 3 months }\end{array}$ & $\begin{array}{c}\text { U for } \\
\text { 3 months }\end{array}$ & $\begin{array}{c}\text { MSE for } \\
\text { 6 months }\end{array}$ & $\begin{array}{c}\text { U for } \\
\text { 6 months }\end{array}$ \\
\hline$(1)$ & $4,931.55$ & 2.0957 & 916.1573 & 0.7356 & $1,585.372$ & 4.1204 \\
$(2)$ & $4,079.66$ & 0.7068 & $3,068.874$ & 1.220 & $2,593.344$ & 3.7704 \\
$(3)$ & $4,069.518$ & 0.6163 & $2,813.607$ & 1.1653 & $2,330.923$ & 3.5238 \\
\hline
\end{tabular}

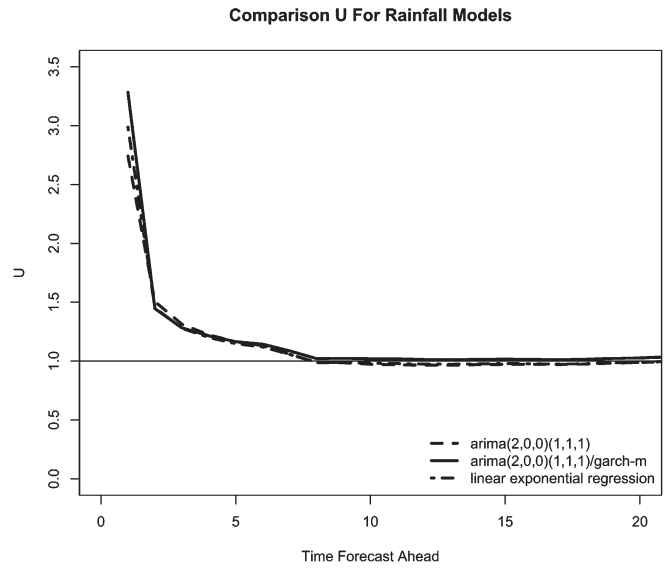

(a)

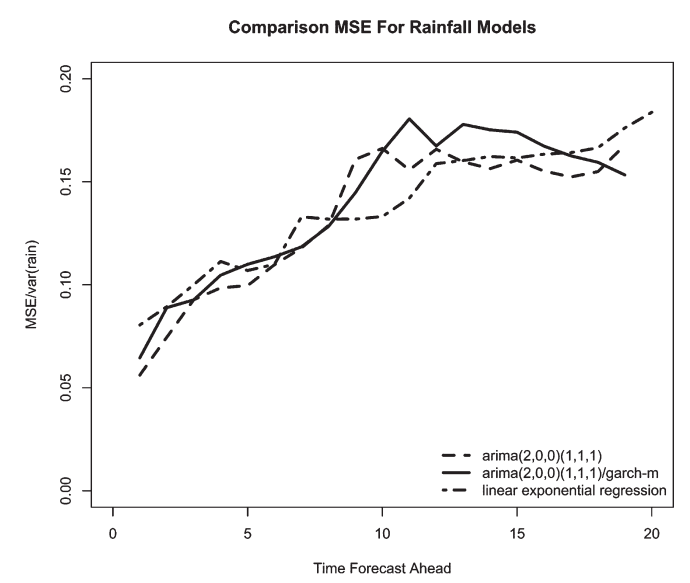

(b)

Figure 12: The (a) mean U and (b) mean MSE of forecasts of rainfall.

\section{CONCLUSION}

Although the SOI and rainfall can be predicted for short terms using time series analysis methods, mean MSE and U values (obtained by repeatedly changing the fitting and testing periods and model estimations) showed that the results in comparison to persistence forecasts are not significantly better. For short term predictions they tend to be worse. This does not mean that these cannot be used but rather it shows that for different fitting and forecast periods, models can fail in predicting ahead. Predicting the rainfall is a complex issue and therefore might be better done by physical in addition to statistical modelling. In fact weather forecasts can predict the short term rainfall well however it cannot predict well the monthly precipitation for two or three or more months ahead. In climate predictions the limit of predictability is reached when the standardised MSE reaches the climate variance (=1). All the rainfall models show an MSE well below 1, i.e. they did not reach the limit of predictability. This paper analysed the relationship between SOI and rainfall as well as predictive errors in statistical modelling. Further research in this area is needed because there is a gap between those short term weather predictions and long term climate forecasts. Our results are in line with Chiew et al. [6] and suggest that the relationship between the SOI and rainfall exists but may not be as strong enough as needed to model the rainfall statistically as a relationship between SOI and rainfall for longer terms forecasting. Clearly, the rainfall in SE QLD may also be influenced more by topography and the seasonal shift of the intertropical convergence zone. 


\section{REFERENCES}

[1] Katz, R.W., Sir Gilbert Walker and a connection between El Nino and statistics. Statistical Science, 17, pp. 97-112, 2002. doi:10.1214/ss/1023799000

[2] Fedorov, A.V., Harper, S.L., Philander, S.G., Winter, B. \& Wittenberg, A., How predictable is El Nino. Bull Am Meteorol Soc, 84, pp. 911-919, 2003. doi:10.1175/BAMS-84-7-911

[3] Ahn, J.H. \& Kim, H.S., Nonlinear modeling of El Nino/southern oscillation index. Journal of Hydrologic Engineering, 10, pp. 8-15, 2005. doi:10.1061/(ASCE)1084-0699(2005)10:1(8)

[4] Latif, M., Anderson, D., Barnett, T., Cane, M., Kleeman, R., Leetmaa, A., O'Brien, J., Rosati, A. \& Schneider, E., A review of the predictability and prediction of ENSO. Journal of Geophysical Research, 103, pp. 14375-14394, 1998. doi:10.1029/97JC03413

[5] Neelin, J.D., Battisti, D.S., Hirst, A.C., Jin, F.F., Wakata, Y., Yamagata, T. \& Zebiak, S.E., ENSO theory. Journal of Geophysical Research C Oceans, 103, p. 14, 1998. doi:10.1029/97JC03424

[6] Chiew, F.H.S., Piechota, T.C., Dracup, J.A. \& McMahon, T.A., El Nino/southern oscillation and Australian rainfall, streamflow and drought: links and potential for forecasting. Journal of Hydrology, 204, pp. 138-149, 1998. doi:10.1016/S0022-1694(97)00121-2

[7] Murphy, B.F. \& Ribbe, J., Variability of southeastern Queensland rainfall and climate indices. International Journal of Climatology, 24, pp. 703-721, 2004. doi:10.1002/joc.1018

[8] Wright, S.J., Carrasco, C., Calderon, O. \& Paton, S., The El Nino southern oscillation, variable fruit production, and famine in a tropical forest. Ecology, 80, pp. 1632-1647, 1999. doi:10.1890/0012-9658(1999)080[1632:TENOSO]2.0.CO;2, doi:10.2307/176552

[9] Podbury, T., Sheales, T.C., Hussain, I. \& Fisher, B.S., Use of El Nino climate forecasts in Australia. American Journal of Agricultural Economics, 80, pp. 1096-1101, 1998. doi:10.2307/1244211

[10] Timmermann, A., Changes of ENSO stability due to greenhouse warming. Geophysical Research Letters, 28, pp. 2061-2064, 2001. doi:10.1029/2001GL012879

[11] Makridakis, S.G., Wheelwright, S.C. \& Hyndman, R.J., Forecasting: Methods and Applications, 3rd edn, Wiley, p. 656, 1997.

[12] Password,F.,Interestrateriskandequity values of Life InsuranceCompanies:aGARCH-Mmodel. Journal of Risk \& Insurance, 74, pp. 401-423, 2007. doi:10.1111/j.1539-6975.2007.00218.x

[13] Zhou, B., He, D. \& Sun, Z., Traffic modeling and prediction using ARIMA/GARCH model. Proc of 2nd EuroNGI Conference on Next Generation Internet Design and Engineering, 2006. 